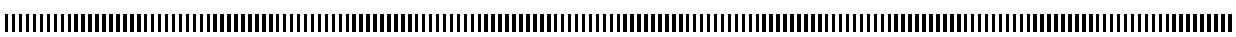

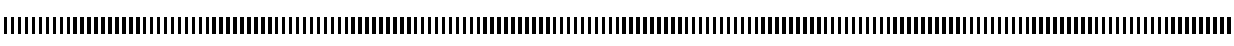

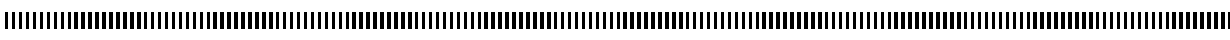

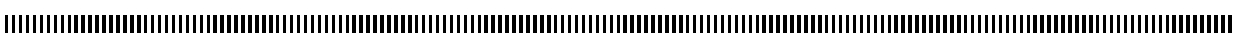

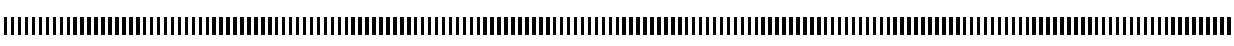

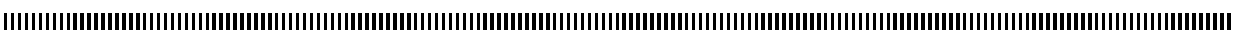

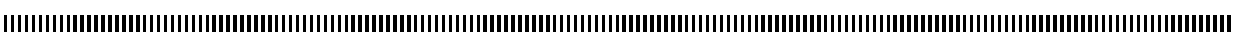

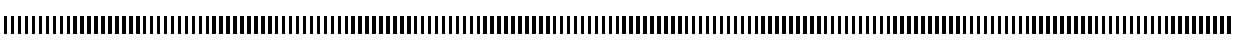

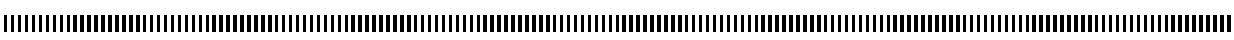

\title{
Nouveau critère de séparation aveugle de sources cyclostationnaires au second ordre
}

\section{New blind separation criterion for second order cyclostationary sources}

\author{
Mohamed Salem OULD MOHAMED ${ }^{1, *}$, Amor KEZIOU $^{2}$, Hassan FENNIRI $^{1}$ \\ et Georges DELAUNAY ${ }^{1}$ \\ ${ }^{1}$ Laboratoire CReSTIC-SYSCOM, Université de Reims \\ 2 FRE 3111 CNRS, Université de Reims et LSTA-Université Paris 6
}

Université de Reims Champagne-Ardenne

UFR Sciences Exactes Naturelles

Moulin de la Housse, BP 1039

51687 REIMS Cedex 2, France

* Corresponding author. Tel.: +33 3269186 00; fax: +33 326913106 .

E-mail address: salem.ould-mohamed@univ-reims.fr (M.S. OULD MOHAMED).

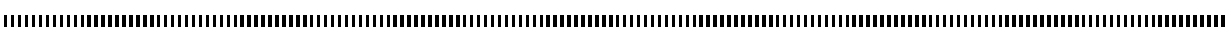

RÉSUMÉ. Dans cet article, nous proposons un nouveau critère de séparation aveugle de sources cyclostationnaires au second ordre de fréquences cycliques supposées connues et toutes différentes, dans le cadre d'un mélange linéaire instantané. La méthode est issue de l'analyse spectrale; elle est basée sur l'exploitation des caractéristiques cyclostationnaires à l'ordre deux des signaux sources. La méthode est facile à mettre en oeuvre. Les résultats théoriques sont présentés et illustrés par des simulations.

ABSTRACT. In this paper, we propose a new blind separation criterion for second order cyclostationary sources with known and different cyclic frequencies. The method results from the spectral analysis; it is based on the use of the second order cyclostationary characteristics of the source signals. The theoretical results are presented, proved and illustrated by simulations.

MOTS-CLÉS : Cyclostationnarité; Séparation aveugle de sources; Statistiques à l'ordre deux; Algorithme du gradient.

KEYWORDS : Cyclostationarity; Blind source separation; Second-order statistic; Gradient algorithm. 


\section{Introduction}

Les premières méthodes de séparation aveugle de sources (SAS) ont été abordées au milieu des années 80 [1]. Comon a ensuite défini le cadre mathématique de l'Analyse en Composantes Indépendantes [2, 3]. La SAS est une technique de traitement du signal qui consiste à restituer un ensemble de signaux non-observables appelés sources à partir d'un ensemble de signaux mesurés appelés observations. La plupart des méthodes de la SAS supposent l'indépendance statistique de celles-ci ; elles consistent à rendre les observations indépendantes au sens statistique. Les différentes méthodes présentées dans la littérature s'appliquent dans le cas stationnaire, certaines de ces méthodes se contentent de rendre les observations indépendantes à l'ordre deux (décorrélation ou blanchiment) $[4,5]$, d'autres utilisent des statistiques d'ordres supérieurs (voir par exemple [6, 7]). Enfin, d'autres méthodes sont basées sur la minimisation de l'Information Mutuelle (voir par exemple $[8,9,10])$. Celles-ci s'appliquent et donnent de bons résultats pour des sources stationnaires ; néanmoins elles ne sont pas généralement exploitables directement dans le cas cyclostationnaire, car les estimateurs statistiques associés ne sont pas généralement adaptés. La SAS cyclostationnaires quant à elle a été étudiée plus récemment ; $[11,12,13,14,16]$ considèrent ce problème et proposent des méthodes s'appliquant dans des situations spécifiques particulières. Castella et al. [17] traitent de la SAS pour des mélanges convolutifs de sources de modulation à phase continue (CPM) qui sont des signaux stationnaires. La difficulté réside dans le fait que ces sources sont non linéaires (et donc non i.i.d). Ils proposent une méthode de séparation "hiérarchique" basée sur la maximisation d'un contraste (qui s'applique dans les deux cas i.i.d et non i.i.d) et qui améliore les méthodes de déflation. La validité de la méthode a été prouvée sous des conditions générales. En outre, Jallon et Chevreuil [15] considèrent la SAS cyclostationnaires ; dans un cadre de sources complexes «circulaires », ils proposent deux versions adaptées de l'algorithme de [3]. Ces versions sont basées sur les cumulants d'ordre quatre et utilisent les auto-corrélations cycliques. La validité des méthodes proposées a été demontrée pour des signaux complexes circulaires sous des conditions généralement vérifiées pour de tels signaux. Bouguerriou et al. [18] proposent une méthode permettant d'extraire un signal d'intérêt cyclostationnaire de fréquence cyclique connue en exploitant l'autocorrélation cyclique dans le cadre d'un mélange instantané. Dans le présent article, nous étendons ces travaux, en proposant une nouvelle méthode de la SAS réelles cyclostationnaires à l'ordre deux de fréquences cycliques connues et distinctes. Cette méthode permet d'extraire simultanément les signaux sources. Les signaux cyclostationnaires sont d'intérêt pratique dans les domaines des radiocommunications, radar, diagnostic des machines tournantes, etc ... Le reste du papier est organisé comme suit. Dans la section 2, nous présentons le modèle de la SAS. Dans la section 3, nous présentons notre critère de séparation, nous montrons que le critère proposé conduit à la séparation et nous prouvons l'existence et l'unicité de la solution. La section 4 est consacrée aux résultats de simulations illustrant la performance de la méthode proposée en comparaison avec l'algorithme proposé dans 
[18].

\section{Modèle de la séparation aveugle de sources}

Le mélange est de la forme

$$
\mathbf{x}=\mathbf{A s}+\mathbf{n}
$$

où $\mathbf{A} \in \mathbb{R}^{p \times p}$ est la matrice de mélange, de dimension $p \times p$ et $\mathbf{s}=\left(s_{1}, \ldots, s_{p}\right)^{T}$ sont les signaux sources, supposés indépendants et cyclostationnaires à l'ordre 2, de fréquences cycliques différentes. $\mathbf{n}$ est le vecteur des signaux bruits, dont les éléments sont supposés centrés, mutuellement indépendants, et indépendants des signaux sources. La présence du bruit additif dans le modèle de mélange complique le problème de la séparation de sources. Pour simplifier le problème, le bruit est supposé négligeable (après pré-traitement). Le but est donc d'estimer les sources $\mathbf{s}$ à partir des observations $\mathbf{x}=$ $\left(x_{1}, \ldots, x_{p}\right)^{T}$. En se restreignant à un estimateur linéaire instantané, nous avons

$$
\mathbf{y}=\mathbf{B x}
$$

où $\mathbf{B} \in \mathbb{R}^{p \times p}$ est la matrice séparante. Le problème consiste donc, à partir des observations $\mathbf{x}$, à déterminer cette matrice $\mathbf{B}$, qui conduit à une bonne estimation des sources $\mathbf{s}$, en exploitant la cyclostationnarité des signaux sources. La méthode que nous proposons se décompose en deux étapes : La première étape consiste à blanchir les observations $\mathbf{x}$. Soit $\mathbf{W} \in \mathbb{R}^{p \times p}$ la matrice de blanchiment des signaux observés $\mathbf{x}$. Notons les signaux blanchis

$$
\mathbf{z}=\mathbf{W} \mathbf{x}
$$

On a donc

$$
\mathbf{z}=\mathbf{W} \mathbf{x}=\mathbf{W A s}
$$

avec $\mathbf{V}:=\mathbf{W A}$ est une matrice unitaire ; c'est-à-dire $\mathbf{V} \mathbf{V}^{\mathbf{T}}=I$ et $\operatorname{det}(\mathbf{V})=1$. Pour retrouver donc les signaux sources $\mathbf{s}$, il faut estimer la matrice inverse $\mathbf{U}$ de $\mathbf{V}$, qui sera par conséquent une matrice unitaire. La deuxième étape consiste à faire une rotation des signaux blanchis $\mathbf{z}$. Soit $\mathbf{U}$ une matrice $p \times p$ telle que $\mathbf{U U}^{\mathbf{T}}=I_{p}$ et $\operatorname{det}(\mathbf{U})=1$. Cette matrice est paramétrée par les rotations de Givens ; elle s'écrit

$$
\mathbf{U}(\theta):=\prod_{1 \leq i<k \leq p} G\left(i, k, \theta_{m}\right)
$$

où 


$$
G\left(i, k, \theta_{m}\right)_{j, l}=\left\{\begin{array}{lll}
\cos \left(\theta_{m}\right) & \text { si } & j=i, l=i \text { ou } j=k, l=k \\
\sin \left(\theta_{m}\right) & \text { si } & j=i, l=k ; \\
-\sin \left(\theta_{m}\right) & \text { si } & j=k, l=i \\
1 & \text { si } & j=l ; \\
0 & \text { sinon, }
\end{array}\right.
$$

pour tous $1 \leq j, l \leq p$, et $\left.\theta_{m} \in\right]-\pi / 2, \pi / 2[, m=1, \ldots, p(p-1) / 2$, sont (les composantes de $\theta$ ) les angles de rotation du plan des observations blanchies $\mathbf{z}$. Le nouveau processus devient

$$
\mathbf{y}=\mathbf{U}(\theta) \mathbf{z} .
$$

La matrice de séparation $\mathbf{B}$ s'écrit donc comme le produit des deux matrices $\mathbf{U}$ et $\mathbf{W}$

$$
\mathbf{B}=\mathbf{U}(\theta) \mathbf{W}
$$

Dans la suite, nous présentons une méthode permettant un choix optimal de $\theta$, basée sur l'utilisation de la corrélation cyclique des sources.

\section{Description de la méthode proposée}

Soit $t$ un instant fixe. Le signal $x(t)$ est cyclostationnaire à l'ordre deux avec des fréquences cycliques $\left(m \alpha_{0} ; m \in \mathbb{Z}\right)$ si sa fonction d'autocorrélation

$$
t \mapsto R_{x}(t, \tau):=\mathbb{E}(x(t) x(t+\tau))
$$

est périodique de période $T_{0}=\frac{1}{\alpha_{0}}$. ( $\mathbb{E}$ désigne ici l'espérance mathématique). Dans ce cas, l'autocorrélation se décompose en série de Fourier comme suit $([19,20])$ :

$$
R_{x}(t, \tau):=\mathbb{E}(x(t) x(t+\tau))=\sum_{m=-\infty}^{+\infty} R_{x}^{m \alpha_{0}}(\tau) \cdot e^{j 2 \pi \frac{m}{T_{0}} t}
$$

avec

$$
R_{x}^{m \alpha_{0}}(\tau)=\lim _{T \rightarrow \infty} \frac{1}{T} \int_{-T / 2}^{T / 2} x(t) x(t+\tau) \cdot e^{-j 2 \pi \frac{m}{T_{0}} t} d t, m \in \mathbb{Z},
$$

si le signal $x(t)$ est "cycloergodique" à l'ordre 2, dans le sens suivant : la moyenne temporelle de

$$
x(t) x(t+\tau) \cdot e^{-j 2 \pi \frac{m}{T_{0}} t}
$$

est un nombre certain. 
Par souci de clarté, et sans perte de généralité, nous présentons la méthode pour un mélange de deux sources $(p=2)$. L'extension au cas de plusieurs sources $(p \geq 3)$ est présentée dans la remarque 2. De l'équation (7), il vient

$$
\begin{aligned}
& y_{1}(t)=\cos (\theta) \cdot z_{1}(t)+\sin (\theta) \cdot z_{2}(t), \\
& y_{2}(t)=-\sin (\theta) \cdot z_{1}(t)+\cos (\theta) \cdot z_{2}(t) .
\end{aligned}
$$

On constate que $y_{1}$ est un mélange de $z_{1}$ et $z_{2}$ qui sont des estimées de $s_{1}$ et $s_{2}$; comme $s_{1}$ et $s_{2}$ sont de fréquences cycliques $\alpha_{1}$ et $\alpha_{2}$ respectivement (avec $\alpha_{1} \neq \alpha_{2}$ ), il est naturel, pour séparer $s_{1}$ de $s_{2}$, de maximiser en $\theta$, l'écart

$$
\Gamma_{1}(\theta)=\sum_{m=1}^{L_{1}}\left(\left|R_{y_{1}}^{m \alpha_{1}}(0)\right|^{2}-\left|R_{y_{1}}^{m \alpha_{2}}(0)\right|^{2}\right) .
$$

De même, comme $y_{2}$ est un mélange de $z_{2}$ et $z_{1}$ qui sont des estimées de $s_{2}$ et $s_{1}$ (respectivement), pour séparer $s_{2}$ de $s_{1}$, on propose de maximiser en $\theta$, l'écart

$$
\Gamma_{2}(\theta)=\sum_{m=1}^{L_{2}}\left(\left|R_{y_{2}}^{m \alpha_{2}}(0)\right|^{2}-\left|R_{y_{2}}^{m \alpha_{1}}(0)\right|^{2}\right) .
$$

Enfin, pour restituer simultanément $s_{1}$ et $s_{2}$ à partir de $y_{1}$ et $y_{2}$, en tenant compte de (11) et (12), on propose de maximiser en $\theta$ le contraste suivant

$$
\begin{gathered}
\Gamma(\theta)=\Gamma_{1}(\theta)+\Gamma_{2}(\theta)=\sum_{m=1}^{L_{1}}\left(\left|R_{y_{1}}^{m \alpha_{1}}(0)\right|^{2}-\left|R_{y_{1}}^{m \alpha_{2}}(0)\right|^{2}\right)+ \\
+\sum_{m=1}^{L_{2}}\left(\left|R_{y_{2}}^{m \alpha_{2}}(0)\right|^{2}-\left|R_{y_{2}}^{m \alpha_{1}}(0)\right|^{2}\right) .
\end{gathered}
$$

Notons que d'après (10), il vient

$$
\begin{aligned}
& R_{y_{1}}^{m \alpha_{1}}(0)=\cos (\theta)^{2} R_{z_{1}}^{m \alpha_{1}}(0)+\sin (\theta)^{2} R_{z_{2}}^{m \alpha_{1}}(0)+2 \cos (\theta) \sin (\theta) R_{z_{1} z_{2}}^{m \alpha_{1}}(0), \\
& R_{y_{1}}^{m \alpha_{2}}(0)=\cos (\theta)^{2} R_{z_{1}}^{m \alpha_{2}}(0)+\sin (\theta)^{2} R_{z_{2}}^{m \alpha_{2}}(0)+2 \cos (\theta) \sin (\theta) R_{z_{1} z_{2}}^{m \alpha_{2}}(0), \\
& R_{y_{2}}^{m \alpha_{2}}(0)=\sin (\theta)^{2} R_{z_{1}}^{m \alpha_{2}}(0)+\cos (\theta)^{2} R_{z_{2}}^{m \alpha_{2}}(0)-2 \sin (\theta) \cos (\theta) R_{z_{1} z_{2}}^{m \alpha_{2}}(0), \\
& R_{y_{2}}^{m \alpha_{1}}(0)=\sin (\theta)^{2} R_{z_{1}}^{m \alpha_{1}}(0)+\cos (\theta)^{2} R_{z_{2}}^{m \alpha_{1}}(0)-2 \sin (\theta) \cos (\theta) R_{z_{1}}^{m \alpha_{2}}(0) .
\end{aligned}
$$

Ces formules sont utilisés pour calculer la fonction coût (13) pour tout $\theta$. Les paramètres $L_{1}$ et $L_{2}$ sont les nombres des coefficients considérés dans la décomposition en série de Fourier de l'autocorrélation et de l'intercorrélation des signaux blanchis $z_{1}$ et $z_{2}$. Le choix de ces paramètres se fait indépendamment de $\theta$ à l'aide d'un seuillage des coefficients des décompositions en série de Fourier de l'autocorrélation et de l'intercorrélation des signaux blanchis $z_{1}$ et $z_{2}$. 
Remarque 1 La méthode proposée reste valable dans le cas où une seule fréquence cyclique est connue (par exemple $\alpha_{1}$ ), si les autres sources sont stationnaires (par exemple $s_{2}$ ). Dans ce cas, nous prenons naturellement $\alpha_{2}=0$, et puisque $L_{1}$ et $L_{2}$ sont des constantes (ne dépendent pas de $\theta$ ), le critère $\Gamma$ peut être simplifié ainsi

$$
\Gamma(\theta)=\sum_{m=1}^{L_{1}}\left|R_{y_{1}}^{m \alpha_{1}}(0)\right|^{2}-\sum_{m=1}^{L_{2}}\left|R_{y_{2}}^{m \alpha_{1}}(0)\right|^{2} .
$$

Remarque 2 La fonction coût $\Gamma$ se généralise au cas de $p$ sources de la manière suivante

$$
\Gamma(\theta)=\sum_{i=1}^{p} \sum_{m=1}^{L_{i}}\left(\left|R_{y_{i}}^{m \alpha_{i}}(0)\right|^{2}-\sum_{j=1 ; j \neq i}^{p}\left|R_{y_{i}}^{m \alpha_{j}}(0)\right|^{2}\right) .
$$

La méthode proposée est justifiée par la proposition suivante (pour la démonstration voir annexe).

Proposition 1 La maximisation du critère $\Gamma$ conduit à la séparation de sources. En outre, la valeur maximale existe et est unique.

Remarque 3 Notons que le critère $\Gamma$ est symétrique et donne les estimations de toutes les sources simultanément.

\section{Résultats de simulations}

Nous présentons dans ce paragraphe deux exemples de simulations pour illustrer la performance de la méthode. Dans le premier exemple, on traite le cas de deux sources cyclostationnaires de fréquences cycliques différentes. Dans le deuxième exemple, nous traitons le cas de trois sources cyclostationnaires de fréquences cycliques toutes différentes.

Exemple 1 Considérons les deux sources cyclostationnaires à l'ordre deux $s_{1}$ et $s_{2}$ respectivement de fréquences cycliques $\alpha_{1}=2 F_{1}=80 \mathrm{~Hz}$ et $\alpha_{2}=2 F_{2}=30 \mathrm{~Hz}$

$$
\begin{aligned}
& s_{1}(t)=a(t) \cos \left(2 \pi F_{1} t\right), \\
& s_{2}(t)=b(t) \cos \left(2 \pi F_{2} t\right),
\end{aligned}
$$

où $a(t)$ et b(t) sont des signaux blancs gaussiens centrés et réduits. Le nombre d'échantillons et la fréquence d'échantillonnage sont fixés respectivement à 4000 et $40 \mathrm{KHz}$. La matrice de mélange est

$$
\mathbf{A}=\left(\begin{array}{cc}
1 & 0.9 \\
0.75 & 1
\end{array}\right)
$$


La figure 1 illustre la courbe representative du critère proposé ; c'est-à-dire la fonction coût

$$
\theta \in] \frac{-\pi}{2}, \frac{\pi}{2}[\mapsto \Gamma(\theta),
$$

le critère proposé dans [18]

$$
\theta \in] \frac{-\pi}{2}, \frac{\pi}{2}\left[\mapsto C_{i}(\theta)=\left|R_{y_{i}}^{\alpha_{i}}(0)\right|^{2}, i=1,2,\right.
$$

et l'erreur quadratique moyenne

$$
\theta \in] \frac{-\pi}{2}, \frac{\pi}{2}\left[\mapsto E Q M(\mathbf{s}, \mathbf{y}, \theta)=\sum_{i=1}^{2}\left\|s_{i}(t)-y_{i}(\theta)\right\|^{2}\right.
$$

entre les signaux sources $\mathbf{s}$ et les estimés y. Dans (14), on choisit $L_{1}=L_{2}=1$; les autres coefficients de la décomposition en série de Fourier sont négligeables. On constate que notre critère est régulier; il admet un seul maximum $\hat{\theta}=-0.0995 \mathrm{rd}$ correspondant à l'estimation optimale (au sens de l'erreur quadratique moyenne) des sources $s_{1}$ et $s_{2}$; (voir figure 1). Les maximums de $C_{1}$ et $C_{2}$ sont respectivement $\hat{\theta_{1}}=-0.0962 \mathrm{rd}$ et $\hat{\theta_{2}}=-0.1027 \mathrm{rd}$. Tous les maximums sont calculés par utilisation de l'algorithme de descente du gradient avec un pas fixe $(\mu=0.01)$, pour 50 itérations, en prenant comme point initial $\theta=0$. Nous observons que le maximum du critère proposé $\Gamma$ est très proche de l'optimum théorique $\widetilde{\theta}=-0.0996 \mathrm{rd}$, qui est le minimum de l'EQM. Pour mesurer la qualité de séparation, nous utilisons le rapport signal sur interference (RSI) défini par

$$
R S I_{i}(\mathbf{s}, \mathbf{y})=10 \log _{10} \frac{E\left\{s_{i}^{2}\right\}}{E\left\{\left(s_{i}-y_{i}\right)^{2}\right\}}, i=1,2 .
$$

Nous présentons dans le tableau 1 , en $\mathrm{dB}$, le RSI pour les trois critères $C_{1}, C_{2}$ et $\Gamma$, où la taille des signaux est $N=4000$. Les simulations sont répétées 500 fois. Les variances des RSIs sont également présentées pour les trois critères. Nous constatons que la méthode proposée donne les meilleurs résultats.

Exemple 2 Considérons les trois sources cyclostationnaires à l'ordre deux de fréquences cycliques respectives $\alpha_{1}=2 F_{1}=30 \mathrm{~Hz}, \alpha_{2}=2 F_{2}=70 \mathrm{~Hz}$ et $\alpha_{3}=2 F_{3}=130 \mathrm{~Hz}$

$$
\begin{aligned}
& s_{1}(t)=a(t) \cos \left(2 \pi F_{1} t\right), \\
& s_{2}(t)=b(t) \cos \left(2 \pi F_{2} t\right), \\
& s_{3}(t)=c(t) \cos \left(2 \pi F_{3} t\right),
\end{aligned}
$$


où $a(t), b(t)$ et $c(t)$ sont des signaux blancs gaussiens centrés et réduits. Le nombre d'échantillons et la fréquence d'échantillonnage sont fixés respectivement à 4000 et 40 Khz. La matrice de mélange est

$$
\mathbf{A}=\left(\begin{array}{ccc}
1 & 0.9 & 0.75 \\
0.75 & 1 & 0.8 \\
0.65 & 0.85 & 1
\end{array}\right)
$$

Dans ce cas $3 \times 3$ de séparation de sources, après l'étape de blanchiment, nous aurons besoin de trois rotations $\theta_{1}, \theta_{2}$ et $\theta_{3}$ de l'espace des signaux blanchis pour pouvoir restituer les sources. Soit $\mathbf{U}$ une matrice $3 \times 3$ telle que $\mathbf{U U}^{\mathbf{T}}=I$ et det $\mathbf{U}=1$. Elle s'écrit

$$
\mathbf{U}(\theta)=U_{1}\left(\theta_{1}\right) \cdot U_{2}\left(\theta_{2}\right) \cdot U_{3}\left(\theta_{3}\right)
$$

où $U_{1}\left(\theta_{1}\right), U_{2}\left(\theta_{2}\right)$ et $U_{3}\left(\theta_{3}\right)$ sont respectivement les rotations de Givens suivantes

$$
\left(\begin{array}{ccc}
\cos \theta_{1} & \sin \theta_{1} & 0 \\
-\sin \theta_{1} & \cos \theta_{1} & 0 \\
0 & 0 & 1
\end{array}\right),\left(\begin{array}{ccc}
\cos \theta_{2} & 0 & \sin \theta_{2} \\
0 & 1 & 0 \\
-\sin \theta_{2} & 0 & \cos \theta_{2}
\end{array}\right),\left(\begin{array}{ccc}
1 & 0 & 0 \\
0 & \cos \theta_{3} & \sin \theta_{3} \\
0 & -\sin \theta_{3} & \cos \theta_{3}
\end{array}\right) .
$$

Les paramètres $\theta_{1}, \theta_{2}$ et $\theta_{3}$ représentent les trois angles de rotation possibles de l'espace des signaux blanchis $\mathbf{z}$, où $-\pi / 2<\theta_{1}, \theta_{2}, \theta_{3}<\pi / 2$. Le critère $\Gamma(\theta)$ dans ce cas s'écrit (voir remarque 2)

$$
\begin{aligned}
\Gamma(\theta)= & \sum_{m=1}^{L_{1}}\left(\left|R_{y_{1}}^{m \alpha_{1}}(0)\right|^{2}-\left(\left|R_{y_{1}}^{m \alpha_{2}}(0)\right|^{2}+\left|R_{y_{1}}^{m \alpha_{3}}(0)\right|^{2}\right)\right)+ \\
& +\sum_{m=1}^{L_{2}}\left(\left|R_{y_{2}}^{m \alpha_{2}}(0)\right|^{2}-\left(\left|R_{y_{2}}^{m \alpha_{1}}(0)\right|^{2}+\left|R_{y_{2}}^{m \alpha_{3}}(0)\right|^{2}\right)\right)+ \\
& +\sum_{m=1}^{L_{2}}\left(\left|R_{y_{3}}^{m \alpha_{3}}(0)\right|^{2}-\left(\left|R_{y_{3}}^{m \alpha_{1}}(0)\right|^{2}+\left|R_{y_{3}}^{m \alpha_{2}}(0)\right|^{2}\right)\right) .
\end{aligned}
$$

Le maximum de la fonction coût $\Gamma$ est calculé par utilisation de l'algorithme de descente du gradient avec un pas fixe $(\mu=0.001)$, pour 100 itérations, en prenant comme point initial $\left(\theta_{1}, \theta_{2}, \theta_{3}\right)=(0,0,0)$. Nous montrons sur le tableau 2 , les RSIs entre les signaux sources et les sources estimées par maximisation de $C_{i}, i=1,2,3$ et $\Gamma$, où la taille des signaux est $N=4000$. Les simulations sont répétées 500 fois. Les variances des RSIs sont également calculées pour les trois critères. Nous présentons dans la figure 2 les signaux sources, les observations et les sources estimés par maximization de $\Gamma$. 


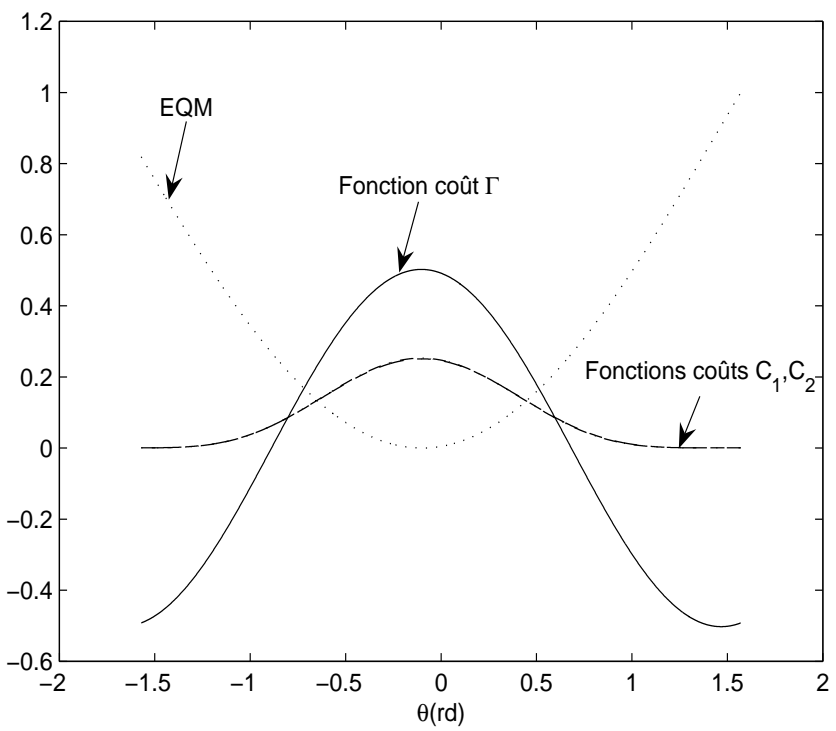

Figure 1. Fonctions coûts $\Gamma, C_{1}, C_{2}$ et Erreur Quadratique Moyenne.

\begin{tabular}{|c|c|c|c|c|c|c|c|}
\hline & $R S I(\mathbf{s}, \mathbf{x})$ & $C_{1}$ & $C_{2}$ & $\Gamma$ & $\sigma_{C_{1}}^{2}$ & $\sigma_{C_{2}}^{2}$ & $\sigma_{\Gamma}^{2}$ \\
\hline$s_{1}$ & 5.45 & 38.78 & -- & 41.08 & 0.177 & -- & 0.160 \\
$s_{2}$ & 6.44 & -- & 39.05 & 41.94 & -- & 0.172 & 0.158 \\
\hline
\end{tabular}

Tableau 1 : RSI en $\mathrm{dB}$ pour $C_{1}, C_{2}$ et $\Gamma$, et leurs variances $\sigma_{C_{1}}^{2}, \sigma_{C_{2}}^{2}$ et $\sigma_{\Gamma}^{2}$.

\begin{tabular}{|c|c|c|c|c|c|c|c|c|c|}
\hline & $R S I(\mathbf{s}, \mathbf{x})$ & $C_{1}$ & $C_{2}$ & $C_{3}$ & $\Gamma$ & $\sigma_{C_{1}}^{2}$ & $\sigma_{C_{2}}^{2}$ & $\sigma_{C_{3}}^{2}$ & $\sigma_{\Gamma}^{2}$ \\
\hline$s_{1}$ & 1.54 & 35.75 & -- & -- & 36.85 & 0.056 & -- & -- & 0.050 \\
$s_{2}$ & 1.85 & -- & 35.30 & -- & 37.20 & -- & 0.067 & -- & 0.060 \\
$s_{3}$ & 1.97 & -- & -- & 35.50 & 37.15 & -- & -- & 0.067 & 0.062 \\
\hline
\end{tabular}

Tableau 2 : RSI en dB pour $C_{1}, C_{2}, C_{3}$ et $\Gamma$, et leurs variances $\sigma_{C_{1}}^{2}, \sigma_{C_{2}}^{2}, \sigma_{C_{3}}^{2}$ et $\sigma_{\Gamma}^{2}$. 

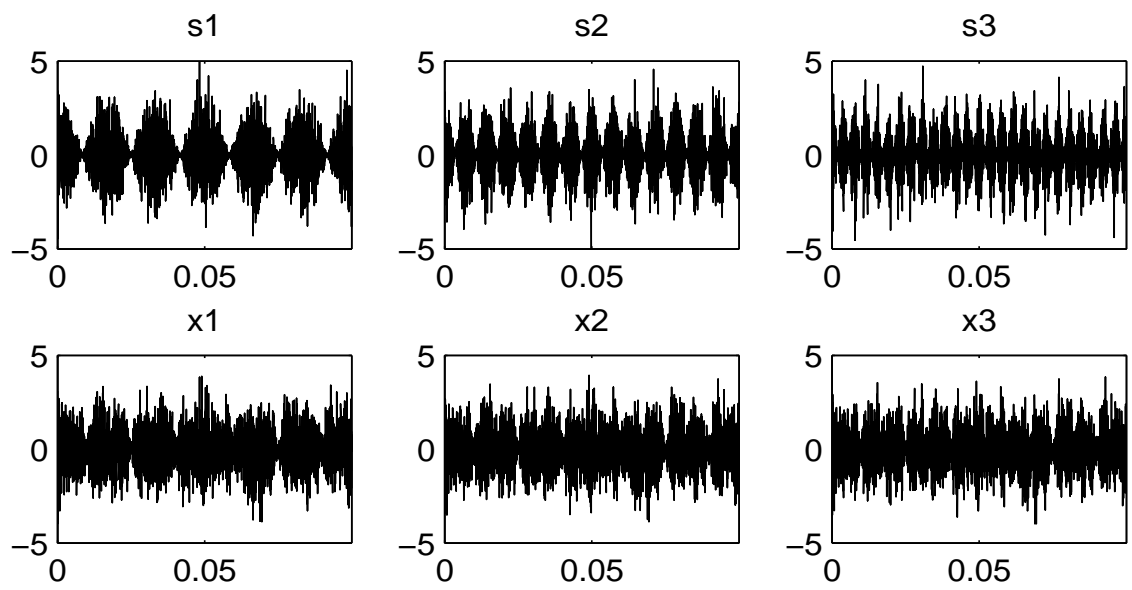

$\mathrm{x} 2$

$\times 3$
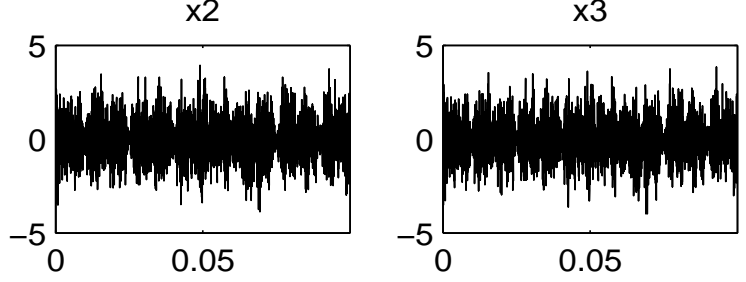

s1 estimé
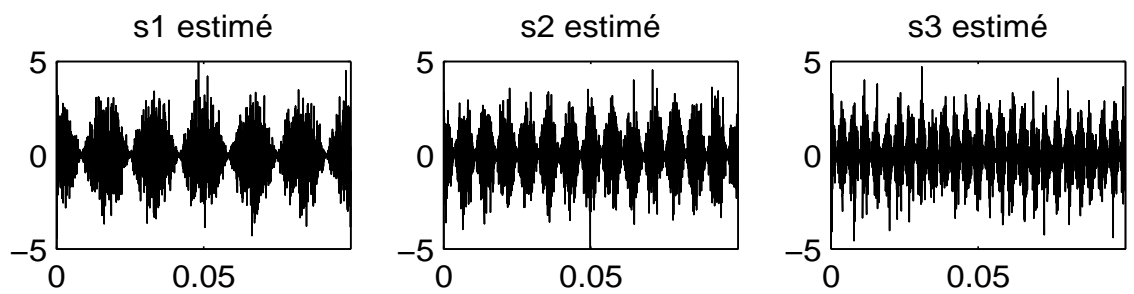

Figure 2. Les signaux sources (en haut), les mélanges (au milieu) et les sources estimées (en bas).

\section{Conclusion}

Dans cet article, nous avons proposé un nouveau critère de la SAS cyclostationnaires au second ordre de fréquences cycliques supposées connues et toutes différentes, dans le cadre d'un mélange linéaire instantané. La méthode exploite les caractéristiques cyclostationnaires à l'ordre deux des signaux sources. La méthode s'applique quel que soit le nombre de sources. Elle est facile à mettre en oeuvre, et peu coûteuse en temps de calcul ; la fonction coût proposée est régulière, elle présente un seul maximum et ce, quelle que soit la dimension du paramètre de rotation. Ceci permet un calcul rapide du maximum par utilisation des algorithmes de type descente du gradient. 


\section{Remerciements}

Les auteurs tiennent à remercier les examinateurs anonymes pour leurs commentaires constructifs et leurs suggestions utiles.

\section{Bibliographie}

[1] Jeanny Hérault, Christian Jutten et B. Ans, « Détection de grandeurs primitives dans un message composite par une architecture de calcul neurominétrique en apprentissage non supervisé », Xème colloque GRETSI, Nice, France, 20-24 May, p. 1017-1022, 1985.

[2] Pierre Comon, «Independent component analysis, a new concept ? », Proc. Int. Workshop on Higher-Order Statistics, Chamrousse, France, p. 111-120, 1991.

[3] Pierre Comon, «Independent component analysis, a new concept? », Signal Processing, IEEE, vol. 36, $\mathrm{n}^{\circ}$ 3, p. 287-314, 1994.

[4] Jean-François Cardoso et Antoine Souloumiac, « Blind signal Beamforming for non Gaussian Signals », Proceedings of the IEEE, vol. 140, $\mathrm{n}^{\circ}$ 6, p. 362-370, 1993.

[5] Adel Belouchrani, Karim Abed-Meraim, Jean-François Cardoso et Eric Moulines, «A Blind Separation Technique Using Second-Order Statistics », IEEE Trans. Signal processing, vol. 45, $\mathrm{n}^{\circ}$ 2, p. 434-444, 1997.

[6] Ali Mansour et Christian Jutten, «Fourth order criteria for blind separation of sources », IEEE Trans on Signal Processing, vol. 43, n 8, p. 2022-2025, August 1995.

[7] ERIC Moreau, «A Generalization of Joint-Diagonalization Criteria for Source Separation », Trans.Signal Processing, IEEE, vol. 49, n 3, p. 530-541, 2001.

[8] Dunh-Tuan Pham, « Mutual Information Approach to blind Separation of stationary sources », IEEE Trans. Information Theory, vol. 48, $\mathrm{n}^{\circ}$ 7, 2002.

[9] Mohammed El Rhabi, Guillaume Gelle, Hassan Fenniri et Georges DelauNAY, «A penalized mutual information criterion for blind separation of convolutive mixtures », Signal Processing, vol. 84, p. 1979-1984, 2004.

[10] Massoud Babaie-Zadeh et Christian Jutten, « A general approach for mutual information minimization and its application to blind source separation », Signal Processing, vol. 85, p. 975-995, 2005.

[11] Anne Ferreol et Pascal Chevalier, « On the behavior of current second and higher order blind source separation methods for cyclostationary sources », IEEE Trans. Signal Processing, vol. 48, p. 1712-1725, June 2000.

[12] Maria. G. Jafari, S. R. Alty et Jonathon A. Chambers, « New natural gradient algorithm for cyclostationary sources », IEEE Proceedings, vol. 151, $\mathrm{n}^{\circ}$ 1, p. 62-68, 2004.

[13] Anne Ferreol, Pascal Chevalier et Laurent Albera, « Second and Higher order blind Separation of first and second order Cylostationary Sources - Application to AM, FSK, CPFSK and Deterministic sources », IEEE Trans. Signal Processing, vol. 52, nº 4, p. 845-861, 
April 2004.

[14] Roger Boustany et Jerome Antoni, « A subspace method for the blind extraction of a cyclostationary source : Application to rolling element bearing diagnostics », Mechanical Systems and Signal Processing, vol. 19, $\mathrm{n}^{\circ}$ 1, p. 1245-1259, 2005.

[15] Pierre Jallon et Antoine Chevreuil, « Separation of instantaneous mixtures of cyclostationary sources », Elsevier. Signal Processing, vol. 87, $\mathrm{n}^{\circ}$ 11, p. 2718-2732, Nov 2007.

[16] Pierre Comon et Christian Jutten, «Séparation de sources, tome 1 :Concepts de base et Analyse en Composantes Independantes », Hermès, série IC2, 2007.

[17] Marc Castella, Pascal Bianchi, Antoine Chevreuil et Jean-Christophe PesQUET, «A blind source separation framework for detecting CPM sources mixed by a convolutive MIMO filter », Elsevier. Signal Processing, vol. 86, p. 1950-1967, 2006.

[18] Noureddine Bouguerriou, Michel Haritopoulos, Cecile Capdessus et Lévi ALlAM, « Novel cyclostationarity-based blind source separation algorithm using second order statistical properties : Theory and application to the bearing defect diagnosis », Mechanical Systems and Signal Processing, vol. 19, $\mathrm{n}^{\circ}$ 6, p. 1260-1281, 2005.

[19] William A. Gardner et Lewis E. Franks, « Characterization of cyclostationary random signal processes », IEEE Trans. Information Theory, vol. 21, $\mathrm{n}^{\circ}$ 1, p. 4-14, 1975.

[20] William A.GARDNER, «Exploitation of the spectral redundancy in cyclostationary signals », IEEE Mag. Signal Processing, vol. 8, 1991.

\section{Annexe}

Démonstration de la Proposition 1. Nous nous restreignons au cas de deux sources $(p=2)$. Le cas de plusieurs sources $(p \geq 3)$ se démontre de manière analogue. Supposons que les signaux sources $s_{1}$ et $s_{2}$ sont centrés, de variance unité et statistiquement indépendants. Les signaux blanchis peuvent être écrits $\mathbf{z}=\mathbf{W} \mathbf{x}=\mathbf{W A s}=\mathbf{V s}$ où $\mathbf{V}=\mathbf{W A}$ est une matrice unitaire, c'est-à-dire, $V V^{T}=I$ et $\operatorname{det}(V)=1$. Elle s'écrit alors sous la forme

$$
\mathbf{V}=\left(\begin{array}{cc}
\cos (\widehat{\alpha}) & \sin (\widehat{\alpha}) \\
-\sin (\widehat{\alpha}) & \cos (\widehat{\alpha})
\end{array}\right)
$$

pour certains $\widehat{\alpha} \in]-\pi / 2, \pi / 2[$. Les signaux estimés sont donc

$$
\mathbf{y}=\mathbf{U z}=\mathbf{U V \mathbf { s }} \text { où } \mathbf{U}=\left(\begin{array}{cc}
\cos (\theta) & \sin (\theta) \\
-\sin (\theta) & \cos (\theta)
\end{array}\right) \text {. }
$$

D'où,

$$
\begin{aligned}
& y_{1}(t)=a(\theta) s_{1}(t)+b(\theta) s_{2}(t) \\
& y_{2}(t)=-b(\theta) s_{1}(t)+a(\theta) s_{2}(t)
\end{aligned}
$$


avec

$$
\begin{aligned}
& a(\theta)=\cos (\theta) \cos (\widehat{\alpha})-\sin (\theta) \sin (\widehat{\alpha}) \\
& b(\theta)=\cos (\theta) \sin (\widehat{\alpha})+\sin (\theta) \cos (\widehat{\alpha}) .
\end{aligned}
$$

La valeur $\widehat{\theta}$ de $\theta$ conduisant à la séparation est telle que $U V=I$, c'est-à-dire, $\widehat{\theta}=-\widehat{\alpha}$. En fait, le calcul direct, montre que $a(\widehat{\theta})=1, \quad b(\widehat{\theta})=0$. Nous montrerons que $\widehat{\theta}=-\widehat{\alpha}$ est l'unique valeur de $\theta$ qui maximise la fonction coût $\Gamma$. En utilisant la formule (18), et le fait que

$$
R_{s_{1}}^{m \alpha_{2}}(0)=R_{s_{2}}^{m \alpha_{1}}(0)=R_{s_{1}, s_{2}}^{m \alpha_{1}}(0)=R_{s_{1}, s_{2}}^{m \alpha_{2}}(0)=0,
$$

nous pouvons écrire le critère sous la forme

$$
\begin{gathered}
\Gamma(\theta)=a(\theta)^{4} \sum_{m=1}^{L_{1}}\left|R_{s_{1}}^{m \alpha_{1}}(0)\right|^{2}-b(\theta)^{4} \sum_{m=1}^{L_{1}}\left|R_{s_{2}}^{m \alpha_{2}}(0)\right|^{2}- \\
b(\theta)^{4} \sum_{m=1}^{L_{2}}\left|R_{s_{1}}^{m \alpha_{1}}(0)\right|^{2}+a(\theta)^{4} \sum_{m=1}^{L_{2}}\left|R_{s_{2}}^{m \alpha_{2}}(0)\right|^{2}
\end{gathered}
$$

soit

$$
\begin{gathered}
\Gamma(\theta)=a(\theta)^{4}\left[\sum_{m=1}^{L_{1}}\left|R_{s_{1}}^{m \alpha_{1}}(0)\right|^{2}+\sum_{m=1}^{L_{2}}\left|R_{s_{2}}^{m \alpha_{2}}(0)\right|^{2}\right]- \\
b(\theta)^{4}\left[\sum_{m=1}^{L_{1}}\left|R_{s_{1}}^{m \alpha_{1}}(0)\right|^{2}+\sum_{m=1}^{L_{2}}\left|R_{s_{2}}^{m \alpha_{2}}(0)\right|^{2}\right] .
\end{gathered}
$$

La dérivée de (20) peut être simplifiée en

$$
\frac{d \Gamma(\theta)}{d \theta}=\left(4 a(\theta)^{3} a^{\prime}(\theta)-4 b(\theta)^{3} b^{\prime}(\theta)\right)\left(\sum_{m=1}^{L_{1}}\left|R_{s_{1}}^{m \alpha_{1}}(0)\right|^{2}+\sum_{m=1}^{L_{2}}\left|R_{s_{2}}^{m \alpha_{2}}(0)\right|^{2}\right) .
$$

Comme

$$
\left(\sum_{m=1}^{L_{1}}\left|R_{s_{1}}^{m \alpha_{1}}(0)\right|^{2}+\sum_{m=1}^{L_{2}}\left|R_{s_{2}}^{m \alpha_{2}}(0)\right|^{2}\right) \neq 0
$$

on peut écrire

$$
\begin{gathered}
\frac{d \Gamma(\theta)}{d \theta}=0 \Longleftrightarrow a(\theta)^{3} a^{\prime}(\theta)-b(\theta)^{3} b^{\prime}(\theta)=0 \Longleftrightarrow \\
\cos (\theta+\widehat{\alpha})=0 \text { ou } \sin (\theta+\widehat{\alpha})=0 .
\end{gathered}
$$


1) $\operatorname{Si} 0<\widehat{\alpha}<\pi / 2,(22) \Longleftrightarrow \theta=-\widehat{\alpha}$ où $\theta=-\widehat{\alpha}+\pi / 2$.

2) $\mathrm{Si}-\pi / 2<\widehat{\alpha}<0,(22) \Longleftrightarrow \theta=-\widehat{\alpha}$ où $\theta=-\widehat{\alpha}-\pi / 2$.

Dans les deux cas, la première solution de $\theta=-\widehat{\alpha}$ est un maximum et le second est un minimum. En fait, un calcul explicite montre que

$$
\left.\frac{d^{2} \Gamma(\theta)}{d \theta^{2}}\right|_{\theta=-\widehat{\alpha}}<0
$$

et

$$
\left.\frac{d^{2} \Gamma(\theta)}{d \theta^{2}}\right|_{\theta=-\widehat{\alpha} \pm \pi / 2}>0
$$

Cela prouve que la fonction coût $\Gamma$ admet un maximum unique $\widehat{\theta}=-\widehat{\alpha}$ qui mène à la parfaite séparation des signaux sources. 